\title{
A report of cherry rusty mottle-associated virus in South Carolina
}

\author{
Bindu Poudel ${ }^{1,2} \cdot$ S. W. Scott ${ }^{1}$
}

Received: 25 January 2017 / Accepted: 14 March 2017 /Published online: 24 March 2017

(C) Australasian Plant Pathology Society Inc. 2017

\begin{abstract}
A cherry tree (Prunus serrulata cv "Shirofugen") exhibiting leaf symptoms frequently associated with viral infection was discovered at the Fruit Research Farm (Musser Farm) of Clemson University. A virus infecting the tree was characterized using molecular techniques, and a BLAST search using the full length genomic sequence of the virus (GenBank Accession KF356396) showed 97\% identity with Cherry rusty mottle-associated virus. This virus had not previously been described from South Carolina. It is suggested that the virus originated from ornamental flowering cherry material ('Yoshino' cherry [Prunus $\times$ yedoensis]) planted on the nearby University Campus and "chip-budded" to the tree of "Shirofugen" as a positive control sample during a bioassay for Prunus necrotic ringspot virus.
\end{abstract}

Keywords Virus $\cdot$ Cherry tree $\cdot$ Sequence $\cdot$ dsRNA .

Rootstock $\cdot$ Scion $\cdot$ TRIFOCAP

In the late summer and early autumn of 2011, suckers growing from the 'Mazzard' cherry F12/1 rootstock (Prunus avium) of a tree of Prunus serrulata cv 'Shirofugen'displayed symptoms of mottling, vein-clearing, and line patterns (Fig. 1). The scion of the tree displayed no symptoms. Symptoms reappeared in the rootstock suckers in 2012 but again no

S. W. Scott

sscott@clemson.edu

1 Department of Biological Sciences, Clemson University, Clemson, SC 29634-0314, USA

2 Present address: IFAS, Tropical Research \& Education Center, Department of Plant Pathology, University of Florida, 18905 SW 280th Street, Homestead, FL 33031-3314, USA symptoms were visible in the scion. To date there had been no reports of symptoms similar to those observed in this tree being described from anywhere in South Carolina. Thus tests were performed to determine the identity of the virus and thereby decide if it posed a threat to other material on the University Fruit Research Farm and to the peach Industry in South Carolina and other southeastern states.

Double-stranded RNA (dsRNA) was extracted from the symptomatic leaf tissue of the rootstock based on the method of Yoshikawa and Converse (1990). Electrophoresis revealed a high molecular weight band of approximately $10 \mathrm{~kb}$. This dsRNA was used in a nested RT-PCR (TriFoCap) assay designed to amplify a conserved region of the polyproteins of viruses in the viral genera Trichovirus, Foveavirus and Capillovirus (Foissac et al. 2005). A fragment, approximately $360 \mathrm{bp}$ in length, was obtained from this assay, cloned into a pGEM®-T Easy Vector (Promega, WI), and sequenced in both directions using M13 forward and M13 reverse primers. The sequence of the amplicon was submitted to the Basic Local Alignment Search Tool (BLAST) search at the National Center for Biotechnology Information (NCBI) and relationships to viruses present in GenBank were identified. cDNA was synthesized using the high molecular weight dsRNA and an anchored oligoDT primer (5' GACCACGC GTATCGATGTCGACTTTTTTTTTTTTTTTTV 3' (Rott and Jelkmann 2001). The region of sequence between the poly A tail of the virus and the TriFoCap amplicon was amplified using the downstream primer 5'GACCACGCGTATCG ATGTCGAC 3' and a primer (5'CGCACATATCATCA CCAGC $3^{\prime}$ ) designed from the sequence of the $360 \mathrm{bp}$ amplicon. A series of RT-PCR reactions involving downstream primers designed from the known developing sequence, and upstream primers designed from the sequences of related viruses present in GenBank, were used to "walk" toward the 5 ' terminus. Contiguous sequence was created by 


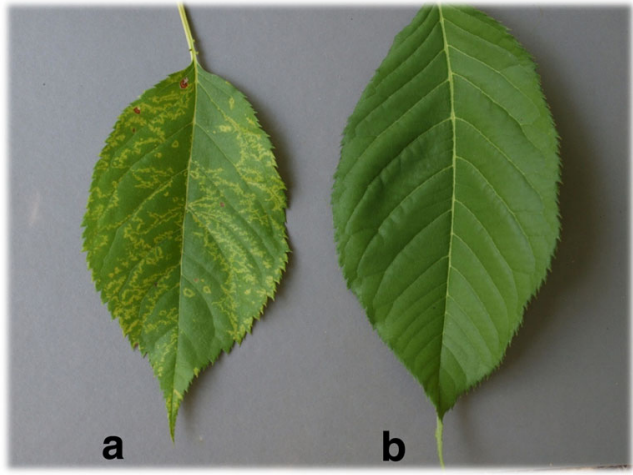

Fig. 1 Virus-like symptoms on the leaves of suckers from a 'Mazzard F12/1' cherry rootstock in 2012 growing at the Clemson University Musser Farm. a: Symptomatic leaves from the sucker of rootstock b: Asymptomatic leaves from the Prunus serrulata cv, 'Shirofugen' scion

joining sequences of PCR fragments using CAP3 (Huang and Madan 1999). Additional PCR reactions that crossed junctions were used to confirm that the assemblies between the fragments were valid and in context. The sequence at the $5^{\prime}$ terminus of the genomic molecule was obtained by using a SMARTer RACE 5'/3' Kit (Takara Bio USA, Inc., Madison WI). All amplicons were cloned into a pGEM®-T Easy Vector and sequenced in both directions using M13 forward and M13 reverse primers.

The sequence of the initial TriFoCap amplicon most closely resembled sequences of Cherry necrotic rusty mottle virus. However, as the genomic sequence was extended it showed the closest match to Cherry rusty mottle-associated virus CRMaV (GenBank Accession KF356396). The complete genomic sequence of this isolate of the virus (CRMaV-SC) has been deposited in GenBank as accession KX389311. The nucleotide sequence of CRMVa-SC is 97\% identical to the sequence of GenBank Accession KF356396 and shares between 75 and $85 \%$ nucleotide identity with the sequences of 7 other isolates of CRMaV currently held in GenBank (October 2016) (KF258176, KF030869, KF030850, KF030849, KC218927, KC218926, and KF030870).

Phylogenetic analysis of the full-length genomic sequences was conducted using MEGA 6 (Tamura et al. 2013). The phylogenetic tree was generated using both a Maximum Likelihood method and Neighbor Joining method with a bootstrap value of 100 replicates. Both methods of tree generation yielded the result in (Fig. 2.). CRMaV-SC is clearly most closely related to isolates of CRMaV (in particular 8804) and therefore should be included in the genus Robigovirus (Villamor et al. 2015) of the family Betaflexiviridae, subfamily Quinvirinae (ICTV 2016). A comparison of the percentage identity of the putative amino acid sequences of the open reading frames (ORF) of CRMVa-SC as compared to the corresponding ORFs of other Robigoviruses is shown in Table 1.
CRMVa-SC is again clearly most closely related to isolate 8804 but is widely divergent in the ORF 1 and the TGB 2 protein in comparison to isolate $\mathrm{S} 83-36$. A relationship clearly supported by the clustering in the phylogenetic tree.

The symptoms observed on the leaves of the P. avium rootstock had not been seen on other trees of the same combination (Shirofugen scion on F12/1 mazzard rootstock) growing at Musser farm. The majority of Trichoviruses, Foveaviruses and Capillovirus have no known vector and although cherry rusty mottle disease has been known for many years there has been no association between the component viruses and an insect vector. The only event in the history of this tree that differed from other trees of the same lineage was that it had been used for a Shirofugen bioassay to detect Prunus necrotic ringspot virus (PNRSV). Buds from a Yoshino' cherry (Prunus $\times$ yedoensis Matsum) tree growing on the campus of Clemson University were "chip-budded" into the Shirofugen scion. The sites at which the buds were inserted were observed for the production of a hypersensitive reaction, indicative of the presence of PNRSV. After the hypersentitive reaction had been noted (approx. 6 weeks after chip-budding) the branch was removed from the tree. A survey (bioassay and PCR) of other Yoshino cherry trees on the campus of the university identified $\mathrm{CRMaV}$ in 4 specimens, including the tree from which the budwood was collected for the Shirofugen bioassay. Although dispersed across campus, the trees had originated from a single batch planted 20-25 years ago. Thus it is speculated that the virus was introduced to the campus over 2 decades ago and was graft-transmitted into the tree displaying symptoms during the bioassay for PNRSV. It is of interest that virus could not be detected in the scion of the tree despite repeated testing using PCR. This would suggest that $P$. serrulata cv Shirofugen is not susceptible to the virus whereas P.serrulata cv Kwanzan, another biological indicator, produced symptoms when graft-inoculated with $\mathrm{CRMaV}$ (Villamor and Eastwell 2013). This is the first report of Cherry rusty mottle-associated virus, in South Carolina. Previous detections of the virus in the US have been reported from the west coast of the USA and with a single exception have all involved infections in $P$. avium. The exception is a detection of the virus in Portuguese laurel (Prunus lusitanica) (Villamor et al. 2014) which is native to southwestern France, Spain, Portugal, and Morocco but has been introduced to the Western US (California, Oregon,and Washington). Clearly, work to determine if peach is indeed a susceptible host, and if so, the potential effects on production, needs to be completed. However, based on the restricted distribution of Portugese laurel and, the relatively low population of cherries (both ornamental and commercial) in the south east, this virus does not appear to pose a threat to the southeastern peach production industry at present. 

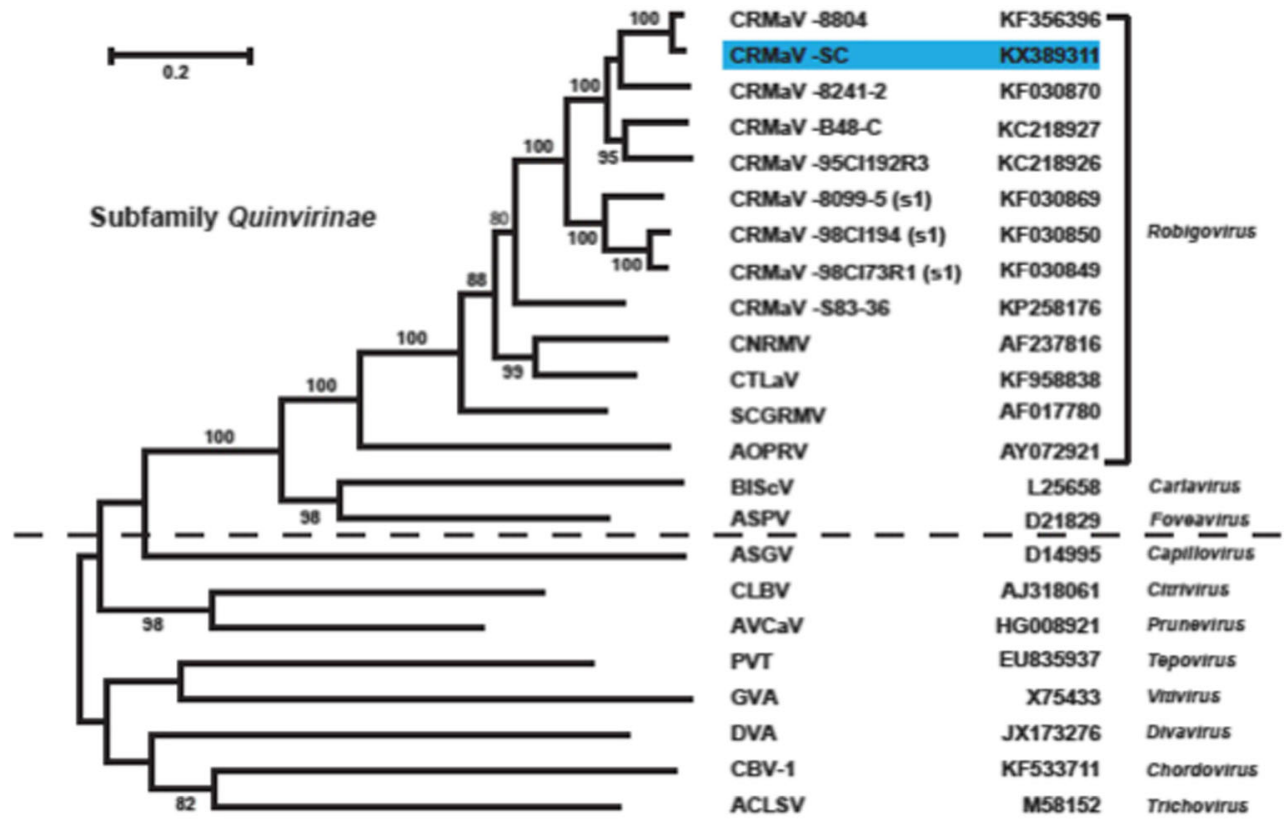

\section{Subfamily Trivirinae}

Fig. 2 Unrooted tree of the family Betaflexiviridae created by using the Maximum Likelihood method based on the Tamura-Nei model (1993) in MEGA6 (2013). The sequence of the isolate described in this manuscript (blue box) and sequences for the type member of each genus contained in the two subfamilies: Quinvirinae and Trivirinae (above and below the dotted line, respectively) are included in addition to the full length genomic sequences for the isolates of Cherry rusty mottle-associated virus currently held in GenBank (December 2016). Bootstrap values were calculated using 100 replications. Bootstrap values of less than 60 are not displayed. Viral acronyms are Cherry rusty mottle-associated virus
(CRMaV), Cherry necrotic rusty mottle virus (CNRMV), Cherry twisted leaf-associated virus (CTLaV), Sour cherry green ring mottle virus (SCGRMV), African oil palm ringspot virus (AOPRV), Blueberry shock virus (B1ScV), Apple stem pitting virus (ASPV), Apple stem grooving virus (ASGV), Citrus leaf blotch virus (CLBV), Apricot vein clearingassociated virus (AVCaV) Potato virus T (PVT), Grapevine virus A (GVA), Diuris virus A (DVA), Carrot Ch Virus 1 (CBV-1) and Apple chlorotic leaf spot virus (ACLSV). Genbank accession numbers are those of sequences used in this work and correspond to the entries used to create the Genome database (Accession Refs NC_XXXXXX) of GenBank

Table 1 The percentage identity of the putative amino acid sequences of the open reading frames (ORF) of CRMVa-SC as compared to the corresponding ORFs of other Robigoviruses. Paired comparisons completed using the EMBOSS needle pairwise sequence alignment for proteins at EMBL-EBI. Viral acronyms correspond to those used in Fig. 2

\begin{tabular}{|c|c|c|c|c|c|c|c|c|}
\hline Virus & Isolate & GenBank Accession & & ORF 1 & TGB 1 & TGB 2 & TGB 3 & $\mathrm{CP}$ \\
\hline CRMaV & 8804 & KF356396 & Robigovirus & 98 & 100 & 98 & 96 & 99 \\
\hline CRMaV & $\mathrm{SC}$ & KX389311 & Robigovirus & 100 & 100 & 100 & 100 & 100 \\
\hline CRMaV & $8241-2$ & KF030870 & Robigovirus & 89 & 94 & 90 & 87 & 96 \\
\hline CRMaV & B48-C & KC218927 & Robigovirus & 91 & 93 & 91 & 90 & 96 \\
\hline CRMaV & 95CI192R3 & KC218926 & Robigovirus & 90 & 94 & 89 & 88 & 94 \\
\hline CRMaV & $8099-5$ (s1) & KF030869 & Robigovirus & 82 & 89 & 86 & 90 & 89 \\
\hline CRMaV & 98CI194 (s1) & KF030850 & Robigovirus & 84 & 89 & 88 & 87 & 90 \\
\hline CRMaV & 98CI73R1 (s1) & KF030849 & Robigovirus & 84 & 88 & 88 & 84 & 90 \\
\hline CRMaV & S83-36 & KP258176 & Robigovirus & 77 & 86 & 68 & 73 & 83 \\
\hline CNRMV & & AF237816 & Robigovirus & 72 & 82 & 55 & 64 & 80 \\
\hline CTLaV & & KF958838 & Robigovirus & 74 & 88 & 68 & 67 & 78 \\
\hline SCGRMV & & AF017780 & Robigovirus & 71 & 79 & 54 & 61 & 74 \\
\hline AOPRV & & AY072921 & Robigovirus & 44 & 52 & 41 & 38 & 41 \\
\hline
\end{tabular}




\section{References}

Foissac X, Svanella-Dumas L, Gentit P, Dulucq M, Armelle Marais A, Candresse T (2005) Polyvalent degenerate oligonucleotides reverse transcription-polymerase chain reaction: a polyvalent detection and characterization tool for Trichoviruses, Capilloviruses, and Foveaviruses. Phytopathology 95:617-625

Huang X, Madan A (1999) CAP3: a DNA sequence assembly program. Genome Res 9:868-877

ICTV (2016) http://ictvonline.Org/taxonomyHistory.Asp?taxnode id=20151662\&taxa_name=Robigovirus. Accessed 10/20/2016

Rott M, Jelkmann W (2001) Complete nucleotide sequence of cherry necrotic rusty mottle virus. Arch Virol 146:395-401

Tamura K, Nei M (1993) Estimation of the number of nucleotide substitutions in the control region of mitochondrial DNA in humans and chimpanzees. Mol Biol Evol 10:512-526
Tamura K, Sketcher G, Filipski A, Kumas S (2013) MEGA6: molecular evolutionary genetics analysis: version 6.0. Mol Biol Evol 30:2725-2729

Villamor DE, Eastwell KC (2013) Viruses associated with rusty mottle and twisted leaf diseases of sweet cherry are distinct species. Phytopathology 103:1287-1295

Villamor DEV, Ward KF, Collman, SJ and Eastwell KC (2014). First Report of Infection of Cherry Rusty Mottle Associated Virus in Portuguese Laurel (Prunus lusitanica) in Washington State Plant Dis. 98, 699

Villamor DEV, Susaimuthu J, Eastwell KC (2015) Genomic analyses of cherry rusty mottle group and cherry twisted leaf-associated viruses reveal a possible new genus within the family Betaflexiviridae. Phytopathology 105:399-408

Yoshikawa N, Converse RH (1990) Strawberry pallidiosis disease: distinctive dsRNA species associated with latent infections in indicators and in diseased strawberry cultivars. Phytopathology 80:543-548 